\title{
Avaliação da contaminação parasitária em folhas de alface da horta ao consumidor
}

\section{final}

\author{
Evaluation of parasitary contamination in lettuce leaves from vegetable garden to final consumer \\ Evaluación de la contaminación parasitaria em hojas de lechuga desde la casa hasta el consumidor
}

final

\author{
Luana Neves Alves \\ ORCID: https://orcid.org/0000-0001-5099-3854 \\ Secretaria Municipal de Saúde Parauapebas, Brasil \\ E-mail: luananevesbiomed@gmail.com \\ Maíra Catherine Pereira Turiel Silva \\ ORCID: https://orcid.org/0000-0002-6460-6154 \\ Universidade Estadual do Pará, Brasil \\ E-mail: mairaturiel@gmail.com \\ Francielle Bonet Ferraz \\ ORCID: https://orcid.org/0000-0002-3489-4781 \\ Universidade Estadual do Pará, Brasil \\ E-mail: francielle.bonet@gmail.com
}

\begin{abstract}
Resumo
A contaminação vegetal pode acontecer em vários processos, desde a irrigação, adubação, coleta, transporte, armazenamento, comercialização e até mesmo a contaminação desencadeada pelas mãos de manipuladores e consumidores. Para minimizar e/ou anular essa contaminação é necessário adesão de medidas que melhorem a higienização do vegetal. Neste estudo foram utilizadas 27 amostras de alface, desfolhadas manualmente e divididas em controle, tratadas com vinagre e tratadas com cloro. Para análise parasitológica foi utilizado o método de sedimentação espontânea. Para análise estatística descritiva foi utilizado os programas Microsoft Excel 10 e para análise estatística inferencial foi utilizado o programa Bioetat $5.0(\mathrm{p} \leq 0,05)$. Todas amostras foram positivas para algum tipo de parasitose em diferentes estágios e graus parasitários. Das 27 amostras, 85,71\% apresentavam parasitas, $67,85 \%$ com organismos potencialmente parasitas e em $100 \%$ deles apresentavam organismos de vida livre. Os vegetais com maior grau parasitário foram os da feira, com 36,95\% de organismos parasitas. Em relação aos tratamentos utilizados, no vinagre foi encontrado um maior número de parasitas, sugerindo que mais organismos se desprenderam da verdura, tornando-a mais adequada ao consumo.
\end{abstract}

Palavras-chave: Vegetais; Contaminação; Parasitose; Sanitizantes.

\begin{abstract}
Vegetable contamination can happen in several processes, from irrigation, fertilization, collection, transportation, storage, marketing and even contamination triggered by the hands of manipulators and consumers. To minimize and / or cancel this contamination, it is necessary to adhere to measures that improve the hygiene of the vegetable. In this study 27 samples of lettuce were used, defoliated manually and divided into control, treated with vinegar and treated with chlorine. For parasitological analysis, the spontaneous sedimentation method was used. The Microsoft Excel 10 programs were used for descriptive statistical analysis and the Bioetat 5.0 program was used for inferential statistical analysis $(\mathrm{p} \leq 0.05)$. All samples were positive for some type of parasitosis in different stages and degrees of parasite. Of the 27 samples, $85.71 \%$ had parasites, $67.85 \%$ with potentially parasitic organisms and $100 \%$ of them had free-living organisms. The vegetables with the highest degree of parasite were those from the fair, with $36.95 \%$ of parasitic organisms. Regarding the treatments used, a greater number of parasites were found in the vinegar, suggesting that more organisms were detached from the vegetable, making it suitable for consumption.
\end{abstract}

Keywords: Vegetables; Contamination; Parasitosis; Sanitizers.

\section{Resumen}

La contaminación vegetal puede ocurrir en varios procesos, desde riego, fertilización, recolección, transporte, almacenamiento, comercialización e incluso contaminación provocada por las manos de manipuladores y consumidores. Para minimizar y / o cancelar esta contaminación, es necesario adherirse a medidas que mejoren la higiene de la verdura. En este estudio se utilizaron 27 muestras de lechuga, defoliadas manualmente y divididas en control, tratadas con vinagre y tratadas con cloro. Para el análisis parasitológico se utilizó el método de sedimentación espontánea. Se utilizaron los programas Microsoft Excel 10 para el análisis estadístico descriptivo y el programa Bioetat 
5.0 para el análisis estadístico inferencial $(\mathrm{p} \leq 0.05)$. Todas las muestras resultaron positivas para algún tipo de parasitosis en diferentes estadios y grados de parásito. De las 27 muestras, el 85,71\% tenían parásitos, el 67,85\% con organismos potencialmente parasitarios y el $100 \%$ de ellos tenían organismos vivos libres. Las hortalizas con mayor grado parasitario fueron las de feria, con un 36,95\% de organismos parasitarios. En relación a los tratamientos utilizados, se encontró una mayor cantidad de parásitos en el vinagre, lo que sugiere que se desprendieron más organismos del vegetal, haciéndolo más apto para el consumo.

Palabras clave: Vegetales; Contaminación; Parasitosis; Desinfectantes.

\section{Introdução}

A alface, conhecida na sociedade científica como Lactuca sativa, integrante da família Asteracea, é considerada a hortaliça mais consumida no Brasil. Esta é a sexta hortaliça em importância econômica e oitava em termos de produção (Fontana, 2008).

Apesar do papel notório das hortaliças consumidas cruas (in natura) para saúde dos consumidores, cabe salientar que estas são grandes veiculadoras de contaminação (Saraiva, et al., 2015) quando higienizadas incorretamente, transmitindo parasitas de diversas classes e provocando inúmeras enfermidades intestinais (Duque, et al., 2014), representando importante problema de saúde pública (Reis, et al., 2007).

As doenças transmitidas por alimentos são resultantes predominantemente do ciclo de contaminação fecal/oral e seu controle tem recebido cada vez maior atenção em todo o mundo. Geralmente, tanto nas áreas rurais quanto urbanas dos países de que ainda possuem más condições sanitárias, as parasitoses intestinais são amplamente difundidas, sendo as hortaliças citadas como um dos veículos de suas estruturas infectantes (Silva, et al., 2011).

A contaminação do vegetal pode acontecer em vários processos desde a irrigação, adubação, coleta, transporte, armazenamento, comercialização até chegar na contaminação desencadeada pelas mãos de manipuladores e consumidores. Além dos vegetais não necessitarem de processamento industrial e nem controles bruscos de temperatura, as hortaliças são mais vulneráveis e propensas a apresentarem diversos tipos de contaminação. A forma inadequada de transporte e armazenamento e, principalmente, de hábitos precários de higiene pessoal e doméstica, compõem os principais fatores que influenciam nesta contaminação e proliferação de microrganismos, entre elas as parasitoses intestinais (De Oliveira, et al, 2014).

Para minimizar ou até anular essa contaminação é necessário adesão de medidas que melhorem a qualidade do vegetal. Os métodos de sanitização geralmente envolvem a aplicação de água, para remover produtos químicos, tratamento mecânico com utilização de escovas ou pulverizadores, seguido de enxágue, sendo que nesta etapa pode ser incluído tratamento sanitizante (Fontana, 2008). Entre os sanitizantes mais usados pelos consumidores pode-se citar o vinagre e cloro, sendo estes agentes frequentemente utilizados por serem considerados eficazes na sanitização de hortaliças e por possuírem baixo valor comercial (Gomes, et al., 2011).

Por questões econômicas, os compostos clorados geralmente são os mais utilizados e amplamente recomendados para retardar ou reduzir o crescimento microbiológico em vegetais minimamente processados (Bachelli, 2010). O cloro, atua como desinfetante através da disrupção da síntese proteica e da permeabilidade da célula do patógeno presente no alimento. O cloro é aceito como desinfetante de utilização segura em vegetais desde 1998, além disso foi estabelecido que após a sua utilização os vegetais devem ser sujeitos a lavagem com água potável (Figueiredo, 2013). O vinagre é utilizado no mundo inteiro como condimento e conservante dos alimentos. Além disso, é considerado complemento indispensável à alimentação humana, pela ação nutritiva e biorregulatória (Soares, 2004).

A Agência Nacional de Vigilância Sanitária (ANVISA) determina, por resolução, que as hortaliças devem ter ausência de sujidades, parasitos e larvas ao serem comercializadas (Quadros, et al., 2011). Contudo, isso não vem sendo seguido como observado por Schemes et al. (2016) em que sua análise, das 45 amostras estudadas, 20 delas (44\%) apresentaram estruturas parasitárias como cistos, larvas e/ou ovos de helmintos em supermercados no sul do Brasil. Da Silva et al. (2017) analisou 48 
amostras, onde 27,1\% (13/48) foram negativas e 72,9\% (35/48) encontraram-se contaminadas por estruturas de parasitos.

Frente a necessidade de investigação das principais fontes de contaminação e dos principais parasitas, e de de uma forma simples e eficaz de sanitização de hortaliças, este estudo investigou parasitas presentes nas alfaces, propondo um método mais eficaz e acessível de higienização.

\section{Metodologia}

Foram analisados 27 pés de alfaces, submetidos a 3 tratamentos cada. Totalizando assim 81 amostras. Foram descartados os alfaces com folhas manchadas e/ou furadas. Raízes foram descartadas.

Cada alface sofreu desfolhação manual e, posteriormente ao desfolhamento, as amostras passaram por divisão manual das folhas, sendo 1/3 para a avaliação sem nenhum tipo de tratamento ou seja, apenas água destilada (controle), os outros $2 / 3$ descendeu para a avaliação com os dois tipos de tratamento: solução de vinagre de álcool (1000:200ml) e solução de cloro 2.000ppm (1000:30ml).

As soluções de cloro e vinagre foram bem espalhadas por sobre todas as superfícies das folhas por 10min. Após esse período, as folhas foram descartadas e o líquido filtrado com gaze de quatro dobras e transportados para cálices parasitológicos de sedimentação por 24h (Gregório, et al., 2012; Velasco, et al., 2014; De Sousa, et al., 2015) sendo utilizado o método de sedimentação espontânea, Hoffman, Pons e Janer (HPJ). Após a sedimentação foram confeccionadas 2 lâminas/amostra, coradas com uma gota de lugol, sobrepostas por lamínula e analisadas em microscópio sob objetiva de 10x e 40x.

Em amostras com presença de larvas foi possível realizar o teste de viabilidade das larvas, ou seja, amostras submetidas a nova análise sem lugol, para confirmar se as mesmas estavam mortas pelo uso do corante ou pela sanitização.

Os dados obtidos foram submetidos ao programa Microsoft Excel 2010 e Bioestat 5.0, onde foram plotados os gráficos e tabelas e, realizados os testes de estatística descritiva por meio de teste qui-quadrado, Teste Ge ANOVA (análise de variância), seguido do Teste de Bonferroni e Tukey para comparar os diferentes grupos de parasitas e tratamentos. Foram considerados significativos valores iguais ou inferiores a $0,05(\mathrm{p} \leq 0,05)$.

\section{Resultados}

A classificação parasitária foi dividida em parasitas, potencialmente parasitas e organismo de vida livre (OVL).

As 81 amostras estiveram positivas para algum tipo de parasitose em diferentes estágios e/ou graus parasitários, onde foi observado uma maior quantidade de organismos de vida livre, sendo 59 estrututras pertencentes a esse grupo. E. Nana e E. coli, ambas com 27 estruturas parasitárias, seguido por ovos de Ancil. sp. com 21 estruturas presentes, G. lamblia com 17, os ovos de Áscaris lumbricoides com 6 e por último com apenas 3 estruturas do protozoário C. mesnili. Totalizando 174 estruturas.

Quando esses dados são separados por estabelecimento onde foram realizadas as coletas, pode-se observar que o local com maior grau parasitário foi a feira onde $36,95 \%$ dos seus organismos, foram considerados parasitas, sendo o supermercado com 27,50\% e por último a horta, onde foi identificado 23,68\% de parasitas. Considerando organismos potencialmente parasitas, encontra-se o supermercado com 27,50\% de todas as estruturas encontradas em suas amostras, seguido pela feira com 19,56\% e a horta com 18,42\%. Em relação a terceira classificação, pode-se observar que dos organismos encontrados na horta, 57,89\% são de vida livre, seguido pela feira com 41,30\% e o supermercado com 45,50\%. (f0,05.,2=-1.5000, p<0.7038). 
Tabela 1 - Frequência relativa e absoluta e desvio padrão dos organismos presentes nas amostras de acordo com a classificação parasitária em cada local de coleta, sem considerar o tratamento.

\begin{tabular}{|c|c|c|c|c|c|c|c|c|c|}
\hline \multirow[b]{2}{*}{ Amostra } & \multicolumn{3}{|c|}{ Supermercado } & \multicolumn{3}{|c|}{ Feira } & \multicolumn{3}{|c|}{ Horta } \\
\hline & Parasito & $\begin{array}{l}\text { Pot. } \\
\text { Parasito } \\
\end{array}$ & OLV & Parasito & $\begin{array}{l}\text { Pot. } \\
\text { Parasito } \\
\end{array}$ & OVL & Parasito & $\begin{array}{l}\text { Pot. } \\
\text { Parasito }\end{array}$ & OLV \\
\hline $\mathbf{1 A}$ & $0,00 \%$ & $18,18 \%$ & $16,67 \%$ & $11,76 \%$ & $11,11 \%$ & $15,79 \%$ & $11,11 \%$ & $28,57 \%$ & $18,18 \%$ \\
\hline 1B & $27,27 \%$ & $18,18 \%$ & $5,56 \%$ & $11,76 \%$ & $11,11 \%$ & $5,26 \%$ & $0,00 \%$ & $0,00 \%$ & $4,55 \%$ \\
\hline $1 \mathrm{C}$ & $0,00 \%$ & $0,00 \%$ & $11,11 \%$ & $11,76 \%$ & $11,11 \%$ & $10,53 \%$ & $22,22 \%$ & $0,00 \%$ & $13,64 \%$ \\
\hline $2 \mathrm{~A}$ & $18,18 \%$ & $9,09 \%$ & $11,11 \%$ & $5,88 \%$ & $11,11 \%$ & $10,53 \%$ & $0,00 \%$ & $28,57 \%$ & $9,09 \%$ \\
\hline 2B & $9,09 \%$ & $27,27 \%$ & $5,56 \%$ & $17,65 \%$ & $22,22 \%$ & $15,79 \%$ & $22,22 \%$ & $14,29 \%$ & $13,64 \%$ \\
\hline $2 \mathrm{C}$ & $9,09 \%$ & $18,18 \%$ & $16,67 \%$ & $17,65 \%$ & $22,22 \%$ & $10,53 \%$ & $11,11 \%$ & $14,29 \%$ & $13,64 \%$ \\
\hline $\mathbf{3 A}$ & $18,18 \%$ & $0,00 \%$ & $11,11 \%$ & $17,65 \%$ & $11,11 \%$ & $15,79 \%$ & $11,11 \%$ & $0,00 \%$ & $9,09 \%$ \\
\hline 3B & $9,09 \%$ & $9,09 \%$ & $11,11 \%$ & $5,88 \%$ & $0,00 \%$ & $10,53 \%$ & $11,11 \%$ & $14,29 \%$ & $13,64 \%$ \\
\hline 3C & $9,09 \%$ & $0,00 \%$ & $11,11 \%$ & $0,00 \%$ & $0,00 \%$ & $5,26 \%$ & $11,11 \%$ & $0,00 \%$ & $4,55 \%$ \\
\hline $\begin{array}{l}\text { Desvio } \\
\text { padrão }\end{array}$ & 01 & 1,09 & 01 & 1,05 & 01 & 0,78 & 01 & 0,83 & 01 \\
\hline
\end{tabular}

Fonte: Autoria própria (2018).

Após o tratamento proveniente das alfaces obtidas do supermercado, foi observado que os OVL encontram-se em maior quantidade, seguido de protozoários e E. coli nos 3 tratamentos (água, vinagre e cloro). Enquanto, nas alfaces provenientes da feira livre, é notória a presença de OVL, contudo, também foi possível observar número maior de ovos de Ancyl. sp. E, nas amostras provenientes da horta, pôde-se observar o constante aparecimento de OVL, seguido pela estrutura parasitária E. Nana (Tabela 2).

Tabela 2 - Frequência relativa e absoluta e desvio padrão dos organismos presentes nas amostras de acordo com a classificação parasitária em cada local de coleta, considerando o tratamento.

\begin{tabular}{|c|c|c|c|c|c|c|c|c|c|c|c|c|c|c|c|c|c|c|}
\hline \multirow[b]{2}{*}{$\begin{array}{l}\text { Formas } \\
\text { evolutivas }\end{array}$} & \multicolumn{6}{|c|}{ Supermercado } & \multicolumn{7}{|c|}{ Feira } & \multicolumn{5}{|c|}{ Horta } \\
\hline & & Água & & Vinagre & & Cloro & & Água & & Vinagre & & Cloro & & Água & & Vinagre & & Cloro \\
\hline $\begin{array}{l}\text { E. nana } \\
\text { Cistos }\end{array}$ & 02 & $11,76 \%$ & 03 & $13,64 \%$ & 02 & $13,33 \%$ & 04 & $16,67 \%$ & 05 & $17,86 \%$ & 02 & $13,33 \%$ & 03 & $13,64 \%$ & 03 & $15,00 \%$ & 03 & $27,27 \%$ \\
\hline $\begin{array}{l}\text { En.coli } \\
\text { Cistos }\end{array}$ & 03 & $17,65 \%$ & 05 & $22,73 \%$ & 04 & $26,67 \%$ & 03 & $12,50 \%$ & 03 & $10,71 \%$ & 02 & $13,33 \%$ & 02 & $9,09 \%$ & 03 & $15,00 \%$ & 02 & $18,18 \%$ \\
\hline $\begin{array}{l}\text { Ancyl sp. } \\
\text { Ovo }\end{array}$ & 00 & $0,00 \%$ & 02 & $9,09 \%$ & 01 & $6,67 \%$ & 03 & $12,50 \%$ & 05 & $17,86 \%$ & 04 & $26,67 \%$ & 01 & $4,55 \%$ & 04 & $20,00 \%$ & 01 & $9,09 \%$ \\
\hline OLV & 10 & $58,82 \%$ & 05 & $22,73 \%$ & 03 & $20,00 \%$ & 11 & $45,83 \%$ & 06 & $21,43 \%$ & 02 & $13,33 \%$ & 14 & $63,64 \%$ & 06 & $30,00 \%$ & 02 & $18,18 \%$ \\
\hline $\begin{array}{l}\text { Nematóide } \\
\text { Adulto }\end{array}$ & 00 & $0,00 \%$ & 02 & $9,09 \%$ & 00 & $0,00 \%$ & 00 & $0,00 \%$ & 04 & $14,29 \%$ & 04 & $26,67 \%$ & 00 & $0,00 \%$ & 02 & $10,00 \%$ & 02 & $18,18 \%$ \\
\hline $\begin{array}{l}\text { G. lamblia } \\
\text { Cisto }\end{array}$ & 01 & $5,88 \%$ & 03 & $13,64 \%$ & 03 & $20,00 \%$ & 02 & $8,33 \%$ & 04 & $14,29 \%$ & 01 & $6,67 \%$ & 01 & $4,55 \%$ & 01 & $5,00 \%$ & 01 & $9,09 \%$ \\
\hline $\begin{array}{l}\text { A. lumbricoides } \\
\text { Ovo }\end{array}$ & 00 & $0,00 \%$ & 02 & $9,09 \%$ & 02 & $13,33 \%$ & 00 & $0,00 \%$ & 01 & $3,57 \%$ & 00 & $0,00 \%$ & 00 & $0,00 \%$ & 01 & $5,00 \%$ & 00 & $0,00 \%$ \\
\hline C.mesnili & 01 & $5,88 \%$ & 00 & $0,00 \%$ & 00 & $0,00 \%$ & 01 & $4,17 \%$ & 00 & $0,00 \%$ & 00 & $0,00 \%$ & 01 & $4,55 \%$ & 00 & $0,00 \%$ & 00 & $0,00 \%$ \\
\hline Total absoluto & 17 & $9,77 \%$ & 22 & 12,64 & 15 & $8,62 \%$ & 24 & $13,79 \%$ & 28 & $16,09 \%$ & 15 & $8,62 \%$ & 22 & $12,64 \%$ & 20 & $11,49 \%$ & 11 & $6,32 \%$ \\
\hline $\begin{array}{l}\text { Total por } \\
\text { Estabelecimento }\end{array}$ & & & $\begin{array}{l}54 \\
31,\end{array}$ & & & & & & & $\begin{array}{l}67 \\
38,50 \%\end{array}$ & & & & & & $\begin{array}{l}53 \\
30,45 \%\end{array}$ & & \\
\hline $\begin{array}{l}\text { Desvio } \\
\text { Padrão }\end{array}$ & 5.8 & & 6.6 & & 4.5 & 826 & & 7.7460 & & 3.3931 & & 4.6098 & & 7.7531 & & 6.1056 & & 3.3582 \\
\hline
\end{tabular}

Fonte: Autoria própria (2018).

Em relação aos tratamentos utilizados, pode-se observar que todos os organismos encontrados nos respectivos tratamentos, sem levar em consideração os estabelecimentos onde as alfaces foram coletadas, os organismos de vida livre foram 
os que apresentaram maior incidência na água, assim como o C. Mesnili. Contudo, a maior quantidade parasitas desprendidos na solução amostral foi encontrada após o tratamento com vinagre, sendo 67 estruturas de parasitas (38,50\%) de um total de 174 estruturas parasitárias encontradas. Deste total, 54 (31,03\%) foram encontradas na água e apenas 53 (30,45\%) no cloro (f0,05.,8 $=0,0273, \mathrm{p}<0,0273 \pm 10.7445)$.

\section{Discussão}

Em coerência com os dados de Quadros et al. (2008), Santos (2012) e Ribeiro et al. (2016) foram analisados de modo que a presença de organismos das diferentes classificações foi equivalente a 100\%, uma vez que todas as amostras estiveram positivas para organismos de vida livre e outros parasitas em uma quantidade menor, porém elevada. Isso significa que não só no município local, mas em diversas regiões do Brasil o parasitismo encontra-se presente contaminando os vegetais, entretanto, sabe-se que a contaminação depende de diversos fatores, como a lavagem, estocagem, transporte, adubo utilizado entre outros meios de contaminação (Gregório, et al., 2012).

Alves et al. (2013) realizaram estudo na cidade de Cuiabá/MT e encontraram contaminação em 66,7\%, já Schemes et al. (2016) em um estudo realizado em 2015 em uma cidade no sul do Brasil, obtiveram resultados positivos em $44 \%$ das amostras. Sendo assim, pode-se perceber que o grau parasitário mesmo quando se trata de baixo número de amostras avaliadas.

Dentre os estabelecimentos estudados neste trabalho, o maior nível de contaminação foi encontrado na feira livre, Em estudos semelhantes, como por Ribeiro et al. (2016), encontrou grau parasitário maior na feira obtendo 56\% das suas amostras positivas e $44 \%$ no supermercado. Tal fato pode ser justificado devido à exposição dos alimentos ao ar livre, manipulação inadequada ou com a poluição urbana e ainda, contando com a possibilidade da maioria dos feirantes possuírem o hábito de lavar as hortaliças, verduras ou frutas em tanques, o que os deixa sujeitos às diversas contaminações, pois nestes locais a água não é trocada com frequência e os recipientes tornam-se cada vez mais sujos (De Sousa, et al., 2015).

Além disso, a falta de higiene pessoal no momento da manipulação dos alimentos também é fator importante na transmissão de enteroparasitas. Indivíduos que manipulam alimentos podem também representar grande fonte de contaminação e disseminação, embora estejam, na maioria das vezes, na condição de portadores assintomáticos de enteroparasitas (Gomes, et al., 2011).

É importante ressaltar que nas amostras pesquisadas foi encontrado multiparasitismo ou seja, mais de um parasita por amostra assim como relatado por Brauer et al. (2016), onde 86,85\% das suas amostras positivas possuíam mais de uma espécie.

Os principais parasitas encontrados nas amostras analisadas foram E. nana, E. coli, Ancil. sp., G.lamblia, A. Lumbricoides e C. mesnili. Tais parasitas também foram encontrados em outros estudos e, podem estar associadas, fundamentalmente, às condições sanitárias do ambiente em que essas amostras são cultivadas (Duarte \& Lacerda, 2014). Moura et al. (2015) relatam um resultado contendo A. lumbricoides (28,5\%), E. coli (10,7\%), Fasciola hepática (3,5\%) e Strongyloides stercoralis $(46,4 \%)$. Tais dados estão em concordância com o presente estudo, apresentando quantidades consideráveis de Ascaris lumbricoides e E. coli. De Sousa et al. (2015), constatou a presença de E. coli (56\%) seguida da presença de E. nana (20\%) e de larvas de helmintos como Strongyloides ssp (50\%).

No presente estudo, pode-se observar a alto incidência do protozoário E. Nana concomitante com E. coli. Ambas as espécies, assim como o C. mesnili são comensais não patogênicas. Estes enterocomensais apresentam o mesmo mecanismo de transmissão de outros protozoários patogênicos como a G. lamblia, indicando portanto que a população está exposta à contaminação fecal, sendo necessária a reflexão sobre as condições sanitárias, práticas de cultivo e manipulação de alimentos, principalmente do ponto de vista higiênico sanitário. Logo, estes enterocomensais são bons indicadores das condições sócio sanitárias e da contaminação fecal a que os indivíduos estão expostos (Moura, 2015).

A baixa prevalência de contaminação por cistos de $C$. mesnili relatada neste estudo corrobora Duarte e Lacerda (2014) 
ao pesquisarem a prevalência de protozoários intestinais em amostras de hortaliças provenientes da região metropolitana do Estado de São Paulo, relataram percentual de contaminação de 4\%. Segundo os autores, embora não patogênico, esse parasita apresenta grande importância como indicador de contaminação fecal de origem humana.

A presença de protozoários de veiculação hídrica como a G. lamblia é coerente, uma vez que as amostras eram provenientes de plantio hidropônico, onde as alfaces são semeadas em estruturas que contenham fluxo contínuo de água, porém nem sempre essa água é tratada da maneira correta. Sabe-se também que mesmo as amostras que são oriundas de plantio tradicional (solo) possuem grande chance de contaminação por meio hídrico, uma vez que são regadas com água contaminada.

Em relação aos helmintos, o parasita mais encontrado foi Ancil. sp, que pode ser explicada devido a contaminação do solo uma vez que era possível no momento da coleta em hortais presenciar a presença de animais como gatos, cachorros, além disso a maioria dos adubos utilizados eram de esterco animal e, de acordo com Stutz et al. (2017). Sendo Marabá/PA uma cidade localizada na região norte, no interior do território amazonense, com clima tropical semiúmido, torna-se ambiente propício para o desenvolvimento de tais parasitas (Brauner, et al., 2016).

Apesar da grande quantidade de ovos de A. lumbricoides nas amostras, os mesmos estavam inviáveis, uma vez que suas membranas estavam rompidas, através do processo químico dos sanitizantes.

Desse modo, em relação aos tratamentos utilizados, constatou-se que nas amostras tratadas com vinagre foi encontrado um maior número de organismos em geral, sendo isso consequência das propriedades químicas do vinagre que consegue diminuir a aderência dos parasitas com os vegetais (Barreto, 2018). Isso sugere que, houve maior a quantidade de parasitas desprendidos, e consequentemente, menor a quantidade desses parasitas nos vegetais durante o consumo. Inclusive, bactérias que outrora era visíveis movimentando-se na amostra controle (com apenas água), nas amostras tratadas com vinagre estas pareciam imóveis. Ainda, cabe salientar que, algumas estruturas maiores também foram visualizadas ao microscópio ótico após o tratamento com vinagre, como ácaros, restos de insetos e até mesmo $C$. mesnile. Sendo assim, o vinagre torna-se uma proposta efetiva e acessível de sanitização dos vegetais.

\section{Conclusões}

Considerando que, todas as amostras possuíam alguma forma parasitária, cabe salientar que o local onde houve maior índice parasitário foram as bancas de feira livre, uma vez que as hortaliças ficam mais expostas ao ambiente aumentando assim o risco de contaminação. Entre todos os métodos de sanitização, conclui-se que o uso do vinagre é de extrema importância, uma vez que o mesmo foi o que mais facilitou o desprendimento das diversas estruturas parasitárias que outrora, estavam presas nas folhas lavadas apenas com água.

Logo, torna-se notória a importância da averiguação das condições sanitárias dos vegetais a serem consumidos juntamente com a caracterização da efetividade dos processos físicos e químicos de desinfecção.

\section{Referências}

Bachelli, M. L. B. (2010). Sanitização para alface minimamente processada em comparação ao hipoclorito de sódio [dissertação]. Campinas: Universidade Estadual de Campinas.

Barreto, N. (2018). Indicadores de qualidade de alfaces (Lactuca sativa L.) produzidas em municípios do sertão paraibano: aspectos microbiológicos e parasitológicos [tese].São Paulo: Universidade de São Paulo.

Brauer, A. M., et al. (2016). Distribuição de enteroparasitos em verduras do comércio alimentício do município de São Mateus, Espírito Santo, Brasil. Natureza Online, 14(1), 55-60.

Da Silva, B., et al. (2017). Avaliação parasitológica de alfaces (lactuca sativa) variedade lisa comercializadas na cidade de manhuaçu-MG. An Sem Cien FACIG, 2(1), 1-7.

De Oliveira, R. M., et al. (2014). Avaliação parasitológica de alfaces (Lactuca sativa) comercializadas no município de Umuarama, Paraná, Brasil. Rev Bras Hig San Anim, 8(3), 1-12. 
Research, Society and Development, v. 10, n. 2, e19410212396, 2021

(CC BY 4.0) | ISSN 2525-3409 | DOI: http://dx.doi.org/10.33448/rsd-v10i2.12396

De Sousa, N., et al. (2015). Avaliação parasitológica de hortaliças: da horta ao consumidor final. Saúde e Pesquisa, 8(2), 255-265.

Duarte, N. E., \& Lacerda, A. F. (2014). Eficiência antimicrobiana e antiparasitária de desinfetantes na higienização de hortaliças na cidade de Natal-RN. Ciên e Nat, 36(2), 92-106.

Duque, I. L., et al. (2014). Pesquisa de ovos de helmintos e oocitos de protozoários em alfaces (Lactuca sativa) comercializadas em feiras livres e supermercados. Vet em Foco, 11(2), 104-111.

Figueiredo, F. F. (2013). Desinfeção de alfaces por ação do cloro e do vinag re e desenvolvimento de um sistema de segurança para alface em estabelecimentos de restauração coletiva [tese]. Lisboa: Universidade Técnica de Lisboa, Faculdade de Medicina Veterinária.

Fontana N. (2006). Atividade antimicrobiana de desinfetantes utilizados na sanitização de alface [monografia]. Santa Maria, Rio Grande do Sul: Centro Universitário Franciscano.

Gomes, C. U. S., et al. (2011). Avaliação das metodologias de higienização de hortaliças in natura empregadas pela população de Medianeira-Pr, utilizando alfaces (lactuca sativa) de diferentes fontes de adubação [monografia]. Medianeira: Universidade Tecnológica Federal do Paraná.

Gregório, D. S., et al. (2012) Estudo da contaminação por parasitas em hortaliças da região leste de São Paulo, São Paulo. Sci Health, 3(2), 96-103.

Moura, L. R. (2015) Pesquisa de parasitos emalface e couve provenientes de feiras da região central e suas mediações na cidade de Anápolis-GO. Rev Educ em Saúde, 3(2), $35-41$.

Quadros, R. M., et al. (2008). Parasitos em alfaces (Lactuca sativa) de mercados e feiras livres de Lages - Santa Catarina. Rev Ciên Saúde, 1(1) 78-84.

Reis, R. M., et al. (2007). Indicador higiênico-parasitário em manipuladores de alimentos em Morrinhos, GO. Est de Biol, 29(68), 313 -317.

Ribeiro, G. M. R., et al. (2016). Avaliação parasitológica de alfaces (Lactuca sativa)comercializadas em feira livre e supermercados na cidade de Muriaé (MG). Rev Cien Faminas, 11(2), 49-57.

Santos, H. S. (2012). Avaliação da eficácia da água sanitária na sanitização de alfaces (Lactuca sativa). Rev Inst Adolfo Lutz, 1(71), 56-60.

Saraiva, N., et al. (2015). Incidência da contaminação parasitária em alfaces nos municípios de Araraquara (SP) e São Carlos (SP). Rev ReBraM , 10(1), 213216.

Schemes, C. M., et al. (2016). Prevalência de parasitos em alfaces (lactuca sativa) de supermercados de uma cidade no sul. Rev Saúde - UNG, 9(3), 18-24.

Silva, J. C., et al. (2011). Parasitismo por Ascaris lumbricoides e seus aspectos epidemiológicos em crianças do estado do Maranhão. Rev Soc Bras Med Trop, 44(1), 100-102.

Soares, B. (2004). Ocorrência de estruturas parasitárias em hortaliças comercializadas em Florianópolis, Santa Catarina [dissertação]. Florianópolis: Universidade Federal de Santa Catarina, Centro de Ciências da Saúde.

Stutz, E. T. G., etal. (2017). Ocorrência de Ancylostoma sp. em fezes de animais coletados empraças e parques públicos do município de Volta Redonda. Episteme Transversallis, 7(2), 9096 .

Velasco, U. P., et al. (2014). Parasitos intestinais em alfaces (Lactuca sativa, l.) das variedades crespa e lisa comercializadas em feiras livres de Niterói-RJ. Rev Pat Trop, 43(2), 209-218. 